\title{
ALCALOIDES INDÓLICOS DE CASCAS DE Aspidosperma vargasii E A. desmanthum
}

\author{
Marycleuma Campos Henrique \\ Departamento de Química, Instituto de Ciências Exatas, Universidade Federal do Amazonas, Av. Rodrigo Octávio Jordão Ramos, \\ 3000, 69077-000 Manaus - AM, Brasil
}

Sergio Massayoshi Nunomura e Adrian Martin Pohlit*

Coordenação de Pesquisa em Produtos Naturais, Instituto Nacional de Pesquisa da Amazônia, Av. André Araújo, 2936, 69060-001

Manaus - AM, Brasil

Recebido em 21/1/09; aceito em 28/7/09; publicado na web em 21/1/10

\begin{abstract}
INDOLE ALKALOIDS FROM THE BARK OF Aspidosperma vargasii AND A. desmanthum. Fractionation of extracts resulted in isolation and identification of indole alkaloids ellipticine and N-methyltetrahydroellipticine from A. vargasii and aspidocarpine from A. desmanthum. Identification of these compounds was achieved based on IR, MS, ${ }^{1} \mathrm{H},{ }^{13} \mathrm{C}$ and 2-D NMR data and comparison to data in the literature.
\end{abstract}

Keywords: Apocynaceae; ellipticine; N-methyltetrahydroellipticine.

\section{INTRODUÇÃO}

O gênero Aspidosperma Mart. pertence à família Apocynaceae ${ }^{1}$ e é distribuído em regiões neotropicais. ${ }^{2}$ Espécies desse gênero são conhecidas popularmente como peroba na maioria das regiões brasileiras e carapanaúba na região amazônica.

A característica marcante desse gênero é a presença de alcaloides indólicos, principalmente os monoterpênicos, ${ }^{3}$ que conferem um amplo espectro de atividades biológicas reconhecidas às espécies desse gênero, tais como antitumoral, ${ }^{4}$ antiplasmódica, ${ }^{5}$ antimicrobiana, ${ }^{6} \mathrm{e}$ antibacteriana $^{7}$ consistentes, em muitos casos, com suas utilizações populares. Dentre essas atividades biológicas, a mais representativa é a citotóxica, que tem sido demonstrada frente a diferentes linhagens de células tumorais. ${ }^{4}$. Este é o caso, por exemplo, da elipticina (1), um dos alcaloides indólicos mais estudados, que chegou a ser utilizada em ensaios clínicos no tratamento do câncer., ${ }^{4,8}$

Este trabalho descreve o isolamento e contribui com dados espectrais mais atuais para a caracterização dos alcaloides elipticina (1) e $\mathrm{N}$-metiltetra-hidroelipticina $\mathbf{2}$ de A. vargasii e aspidocarpina (3) de A. desmanthum (Figura 1).

\section{PARTE EXPERIMENTAL}

\section{Instrumentação e material}

Os espectros de infravermelho (IV) foram obtidos em um aparelho FTLA 2000-104 da Bomem, utilizando-se pastilhas de $\mathrm{KBr}$, com frequências medidas em $\mathrm{cm}^{-1}$. Os espectros de RMN foram obtidos em $\mathrm{CDCl}_{3}$ ou $\mathrm{CD}_{3} \mathrm{OD}$ em um aparelho Unity Inova da Varian $\left({ }^{1} \mathrm{H}\right.$ : $\left.500 \mathrm{MHz} ;{ }^{13} \mathrm{C}: 125 \mathrm{MHz}\right)$. Espectros de massas com ionização por electrospray de alta resolução (ESI-HR-MS) foram obtidos em um Bruker-Daltronics UltraOTof em modo positivo e negativo utilizando $\mathrm{H}_{2} \mathrm{O} / \mathrm{MeOH}$ como solventes de infusão. Para CCD foram utilizadas sílica gel 60 e sílica gel 60 RP-18 (Merck). Para as colunas cromatográficas foram utilizadas sílica gel 60 (40$63 \mu \mathrm{m})$, sílica gel $60(63-200 \mu \mathrm{m})$ e sílica gel 60 RP-18 (40-63 $\mu \mathrm{m})$ da Merck. Em CCD, as substâncias foram evidenciadas pelo uso de radiação ultravioleta (UV) sob comprimentos de onda de 254 e $366 \mathrm{~nm}$, pela impregnação das placas em cubas de vidro saturadas

\footnotetext{
*e-mail: ampohlit@inpa.gov.br
}

por vapores de iodo, anisaldeído e reagente de Dragendorff (para revelação de compostos nitrogenados).
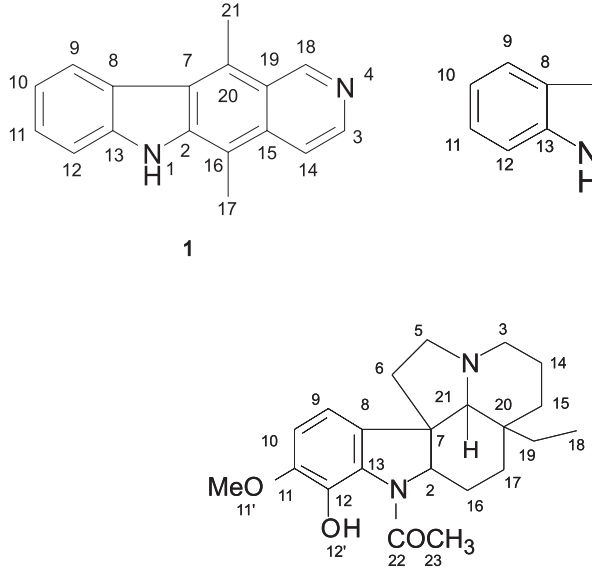

Figura 1. Estruturas dos alcaloides indólicos (1-3) isolados de das cascas de A. vargasii e A. desmanthum

\section{Material vegetal}

As cascas das Aspidosperma foram coletadas na Reserva Adolpho Ducke, no km 26 da rodovia Manaus-Itacoatiara a partir de espécimes previamente identificados no Projeto Flora da Reserva Ducke. Exsicatas número 180459 para $A$. desmanthum e 191853 para A. vargasii estão depositadas no herbário do Instituto Nacional de Pesquisa da Amazônia (INPA).

\section{Extração e isolamento dos constituintes químicos}

Aspidosperma vargasii $A$. DC.

O extrato alcaloídico de A. vargasii A. DC. foi obtido através de maceração contínua das serragens das cascas $(1,30 \mathrm{~kg})$ com solução $\mathrm{EtOH} / \mathrm{NH}_{4} \mathrm{OH} 1 \%$ à temperatura ambiente em um frasco mariote, deixando-se repousar por 7 dias com agitação diária. A mistura foi filtrada e o procedimento repetido por quatro vezes consecutivas. As soluções obtidas foram reunidas e o solvente foi eliminado utilizando-se rotaevaporador. A mistura final foi submetida à liofilização, 
originando o extrato EtOH/ $/ \mathrm{NH}_{4} \mathrm{OH} 1 \%$ (56 g). O extrato alcaloídico foi dissolvido em $100 \mathrm{~mL}$ de AcOEt e, em seguida, acidulado com solução de $\mathrm{HCl} \mathrm{0,1} \mathrm{N}$. A seguir, foi feito fracionamento para extração de alcaloides ajustando-se o pH da fração aquosa ácida em 8,0 com adição $\mathrm{NH}_{4} \mathrm{OH}$ concentrada e extraindo-se com $100 \mathrm{~mL}$ de $\mathrm{CHCl}_{3}$ por três vezes. Em seguida, a fração aquosa com $\mathrm{pH}$ 8,0 teve seu $\mathrm{pH}$ elevado a 10 por adição de $\mathrm{NaHCO}_{3}$ e foi extraída com $100 \mathrm{~mL}$ de $\mathrm{CHCl}_{3}$ por três vezes. Por último, o $\mathrm{pH}$ da fração aquosa foi ajustado em 12 com adição de solução de $\mathrm{NaOH} 0,1 \mathrm{~N}$ e foi extraída com $100 \mathrm{~mL}$ de $\mathrm{CHCl}_{3}$ por três vezes. Os solventes foram eliminados das frações em um rotaevaporador. Ao final do procedimento, foram obtidas as frações AcOEt (1,06 g), clorofórmicas em pH 8 (8,03 g), $10(2,8 \mathrm{~g}), 12(0,46 \mathrm{~g})$ e aquosa $(3,45 \mathrm{~g})$.

A fração clorofórmica $\mathrm{pH} 8(7,0 \mathrm{~g})$ de $A$. vargasii foi submetida à cromatografia em coluna de sílica gel 60 Merck (40-63 $\mu \mathrm{m})$ eluindo-se com $\mathrm{CHCl}_{3} / \mathrm{MeOH}$ em gradiente crescente de polaridade e resultando em 44 frações de $50 \mathrm{~mL}$ cada. Em seguida, as amostras obtidas foram analisadas em CCD e reunidas de acordo com sua similaridade em 3 grupos (G1-G3). A fração reunida G3 (3,99 g) foi fracionada em CC de sílica gel (40-63 $\mu \mathrm{m})$, utilizando-se acetona/ $i$-PrOH com $1 \%$ de $\mathrm{Et}_{2} \mathrm{NH}$ com aumento de polaridade, obtendo-se 206 frações de aproximadamente $10 \mathrm{~mL}$ cada, as quais foram reunidas conforme a similaridade em 4 grupos (A1-A4). A fração A2 (93,0 mg) foi purificada por CCDP (sílica gel $60 \mathrm{PF}_{254}$ ) utilizando-se como eluente $100 \mathrm{~mL}$ da mistura $\mathrm{CH}_{2} \mathrm{Cl}_{2} / \mathrm{MeOH}$ (8:2) obtendo-se a elipticina (1, $68,3 \mathrm{mg}$, p.f. $311-13^{\circ} \mathrm{C}$ ) em forma de cristais amarelos pontiagudos. A fração reunida $\mathrm{G} 4$ forneceu por CCDP (sílica gel $60 \mathrm{PF}_{254}$ ) a Nmetiltetra-hidroelipticina (2, 55,3 mg, p.f. $\left.218-223^{\circ} \mathrm{C}\right)$, um sólido amorfo amarelo, em eluição com $100 \mathrm{~mL}$ da mistura de $\mathrm{CH}_{2} \mathrm{Cl}_{2} /$ $\mathrm{MeOH}$ com $1 \%$ de $\mathrm{Et}_{2} \mathrm{NH}$.

\section{Aspidosperma desmanthum Benth ex. Mull}

A serragem da casca do tronco $(1,20 \mathrm{~kg})$ foi submetida à extração, semelhante à descrita acima, e forneceu o extrato alcaloídico (35,98 g), e após extração ácido-base forneceu cinco frações: AcOEt (2,03 g), $\mathrm{CHCl}_{3} \mathrm{pH} 8(1,45 \mathrm{~g}), \mathrm{CHCl}_{3} \mathrm{pH} 10$ (0,22 g), $\mathrm{CHCl}_{3} \mathrm{pH} 12$ $(0,40 \mathrm{~g})$ e aquosa $(4,37 \mathrm{~g})$ A fração clorofórmica $\mathrm{pH} 8(1,4 \mathrm{~g})$ foi fracionada em coluna de sílica gel $(40-63 \mu \mathrm{m})$ e eluída com mistura de solventes $\mathrm{CH}_{2} \mathrm{Cl}_{2} / \mathrm{MeOH}$, obtendo-se 82 frações de $50 \mathrm{~mL}$, que foram reunidas de acordo com suas similaridades em 7 grupos (M1M7). A fração M4 foi re-fracionada em CC de sílica gel (40-63 $\mu \mathrm{m})$, utilizando-se como eluente $\mathrm{CH}_{2} \mathrm{Cl}_{2} / i-\mathrm{PrOH}$, obtendo-se 40 frações de aproximadamente $40 \mathrm{~mL}$ e foram reunidas em 4 grupos (S1-S4). A fração $\mathrm{S} 4$ forneceu a aspidocarpina $\left(\mathbf{3}, 68,5 \mathrm{mg}\right.$, p.f. $\left.311-13^{\circ} \mathrm{C}\right)$ um sólido cristalino branco.

Elipticina (1): cristais amarelos pontiagudos, p.f. $311-13^{\circ} \mathrm{C}$ (lit.: $\left.315-17{ }^{\circ} \mathrm{C}\right),{ }^{9} \mathrm{RMN}{ }^{1} \mathrm{H}\left(500 \mathrm{MHz}, \mathrm{CDCl}_{3}: \mathrm{CD}_{3} \mathrm{OD}, 1: 1\right): 3 \mathrm{H}-17\left(\delta_{\mathrm{H}}\right.$ $2,7, \mathrm{~s}), 3 \mathrm{H}-21\left(\delta_{\mathrm{H}} 3,2, \mathrm{~s}\right), 1 \mathrm{H}-10\left(\delta_{\mathrm{H}} 7,2, \mathrm{ddd}=7,5 ; 6,8 ; 1,5\right), 2 \mathrm{H}-11 \mathrm{e}$ $12\left(\delta_{\mathrm{H}} 7,4 \mathrm{a} 7,5, \mathrm{~m}\right), 1 \mathrm{H}-14\left(\delta_{\mathrm{H}} 7,9, \mathrm{~d}=7,0\right), 1 \mathrm{H}-9\left(\delta_{\mathrm{H}} 8,3, \mathrm{~d}=7,0\right)$, $1 \mathrm{H}-3\left(\delta_{\mathrm{H}} 8,3, \mathrm{~d}=7,0\right), 1 \mathrm{H}-18\left(\delta_{\mathrm{H}} 9,5, \mathrm{~s}\right) . \mathrm{RMN}{ }^{13} \mathrm{C} \quad(125 \mathrm{MHz}$, $\left.\mathrm{CDCl}_{3}: \mathrm{CD}_{3} \mathrm{OD}\right): \mathrm{C}-18\left(\delta_{c} 148,3\right), \mathrm{C}-13\left(\delta_{c} 143,1\right), \mathrm{C}-2\left(\delta_{c} 141,9\right)$, $\mathrm{C}-3\left(\delta_{c} 137,6\right), \mathrm{C}-15\left(\delta_{c} 133,4\right), \mathrm{C}-20\left(\delta_{c} 129,0\right), \mathrm{C}-11\left(\delta_{c} 127,3, \mathrm{C}-7\right.$ $\left(\delta_{c} 124,8, \mathrm{C}-9\left(\delta_{c} 123,8\right), \mathrm{C}-8\left(\delta_{c} 123,6\right), \mathrm{C}-19\left(\delta_{c} 122,2\right), \mathrm{C}-10\left(\delta_{c}\right.\right.$ $119,5), \mathrm{C}-14\left(\delta_{c} 116,9\right), \mathrm{C}-12\left(\delta_{c}^{c} 110,7\right), \mathrm{C}-16\left(\delta_{c}^{c} 108,4\right), \mathrm{C}-21\left(\delta_{c}^{c}\right.$ $14,1), \mathrm{C}-17\left(\delta_{\mathrm{c}} 11,2\right)$.

N-metiltetra-hidroelipticina 2: um sólido amorfo amarelo, p.f. 218$223{ }^{\circ} \mathrm{C}$ (lit.: $\left.215-225^{\circ} \mathrm{C}\right),{ }^{10} \mathrm{RMN}{ }^{1} \mathrm{H}\left(500 \mathrm{MHz}, \mathrm{CDCl}_{3}: \mathrm{CD}_{3} \mathrm{OD}\right)$ : $3 \mathrm{H}-17\left(\delta_{\mathrm{H}} 2,4, \mathrm{~s}\right), 3 \mathrm{H}-4$ ' $\left(\delta_{\mathrm{H}} 2,6, \mathrm{~s}\right), 3 \mathrm{H}-21\left(\delta_{\mathrm{H}} 2,7, \mathrm{~s}\right), 2 \mathrm{H}-3\left(\delta_{\mathrm{H}} 2,8\right.$, $\mathrm{t}=6,5), 2 \mathrm{H}-14\left(\delta_{\mathrm{H}} 3,0, \mathrm{t}=6,5\right), 2 \mathrm{H}-18(3,8, \mathrm{~s}), 1 \mathrm{H}-10\left(\delta_{\mathrm{H}} 7,1, \mathrm{t}=7,8\right)$, $1 \mathrm{H}-11\left(\delta_{\mathrm{H}} 7,3, \mathrm{t}=7,8\right), 1 \mathrm{H}-12\left(\delta_{\mathrm{H}} 7,4, \mathrm{~d}=7,8\right), 1 \mathrm{H}-9\left(\delta_{\mathrm{H}} 8,2, \mathrm{~d}=7,8\right)$. $\mathrm{RMN}{ }^{13} \mathrm{C}\left(125 \mathrm{MHz}, \mathrm{CDCl}_{3}: \mathrm{CD}_{3} \mathrm{OD}\right): \mathrm{C}-13\left(\delta_{\mathrm{c}} 140,8\right), \mathrm{C}-2\left(\delta_{\mathrm{c}} 138,7\right)$, $\mathrm{C}-15(\delta 128,6), \mathrm{C}-20(\delta 126,3), \mathrm{C}-11(\delta$ 124,6), C-8 $(\delta$ 124,1), C-9 $\left(\delta_{c} 122,3\right), \mathrm{C}-19\left(\delta_{c} 122,2\right), \mathrm{C}-7\left(\delta_{c} 120,1\right), \mathrm{C}-10\left(\delta_{c} 118,4\right), \mathrm{C}-16\left(\delta_{c}\right.$
$114,9), \mathrm{C}-12\left(\delta_{c} 110,6\right),-18\left(\delta_{c} 56,5\right), \mathrm{C}-3\left(\delta_{c} 52,5\right), \mathrm{C}-4{ }^{\prime}\left(\delta_{c} 45,4\right)$, $\mathrm{C}-14\left(\delta_{c} 27,3\right), \stackrel{\mathrm{C}}{\mathrm{C}}-21\left(\delta_{c} 14,7\right), \stackrel{\mathrm{C}}{\mathrm{C}}-17\left(\delta_{c} 12,1\right)$.

Aspidoscarpina (3): sólido cristalino branco, p.f. $169-171{ }^{\circ} \mathrm{C}$ (lit.: $\left.166-169^{\circ} \mathrm{C}\right),{ }^{11} \mathrm{RMN}{ }^{1} \mathrm{H}\left(500 \mathrm{MHz}, \mathrm{CDCl}_{3}\right): 1 \mathrm{H}-18\left(\delta_{\mathrm{H}} 0,6, \mathrm{t}=7,5\right)$, $1 \mathrm{H}-19\left(\delta_{\mathrm{H}} 0,9, \mathrm{dq}=14 ; 7\right), 1 \mathrm{H}-15^{\prime}\left(\delta_{\mathrm{H}} 1,1, \mathrm{dd}=13,5 ; 4,5\right), 1 \mathrm{H}-14^{\prime}(1,1$ , d =13,5), 1H-19' $\left(\delta_{\mathrm{H}} 1,4, \mathrm{dq}=14 ; 7\right), 4 \mathrm{H}-16 ; 6^{\prime} ; 15 ; 17\left(\delta_{\mathrm{H}} 1,5-1,7\right.$, $\mathrm{m}), 1 \mathrm{H}-17^{\prime}\left(\delta_{\mathrm{H}} 1,7, \mathrm{dd}=13 ; 3,5\right), 1 \mathrm{H}-16^{\prime}\left(\delta_{\mathrm{H}} 1,8-1,9, \mathrm{~m}\right), 3 \mathrm{H}-\left(\delta_{\mathrm{H}}\right.$ $1,9, \mathrm{~m}), 1 \mathrm{H}-21\left(\delta_{\mathrm{H}} 2,2, \mathrm{~s}\right), 1 \mathrm{H}-5,\left(\delta_{\mathrm{H}} 2,3, \mathrm{~d}=9\right), 1 \mathrm{H}-23\left(\delta_{\mathrm{H}} 2,3, \mathrm{~s}\right)$, $1 \mathrm{H}-3\left(\delta_{\mathrm{H}} 3,0, \mathrm{~d}=3,5\right), 1 \mathrm{H}-5\left(\delta_{\mathrm{H}} 3,1, \mathrm{ddd}=9 ; 9 ; 2\right), 1 \mathrm{H}-11^{\prime}\left(\delta_{\mathrm{H}} 3,8, \mathrm{~s}\right)$, $1 \mathrm{H}-2\left(\delta_{\mathrm{H}} 4,1, \mathrm{dd}=11 ; 6\right), 1 \mathrm{H}-9\left(\delta_{\mathrm{H}} 6,6, \mathrm{~d}=8,0\right), 1 \mathrm{H}-10\left(\delta_{\mathrm{H}} 6,7, \mathrm{~d}=8\right)$, $1 \mathrm{H}-12$ ' $\left(\delta_{\mathrm{H}} 10,9, \mathrm{~s}\right) . \mathrm{RMN}{ }^{13} \mathrm{C}\left(125 \mathrm{MHz}, \mathrm{CDCl}_{3}\right): \delta 169,5,149,6$, $137,8,133,3,127,7,112,6,110,3,70,9,70,5,53,9,53,7,52,7,52,4$, $39,6,35,7,34,3,30,3,25,4,23,2,22,9,21,8,7,0$.

\section{RESULTADOS E DISCUSSÃO}

Na Amazônia muitas espécies de Aspidosperma são utilizadas pelas populações indígena e cabocla por suas propriedades medicinais. A infusão da casca de algumas espécies, como A. nitidum e A. album, A. discolor, A. excelsum e A. polineuron, é utilizada no tratamento de malária. ${ }^{12,13}$ A A. nitidum é utilizada no tratamento do útero, ovário, câncer, diabetes, estômago e como anticonceptivo. ${ }^{14}$ Extrato alcaloídico das cascas de $A$. ramiflorum demonstrou eficácia no tratamento de leishmaniose $\mathrm{e}^{15}$ e foi demonstrada atividade antimalárica em extratos polares das cascas de A. megalocarpon. ${ }^{16}$ A presença de alcaloides indólicos nas duas espécies em estudo já era esperada, uma vez que as espécies de Aspidosperma são caracterizadas pela ocorrência frequente de substâncias alcaloídicas, tornando-se bons marcadores quimiotaxonômicos para classificação dessas espécies. ${ }^{17} \mathrm{O}$ fracionamento cromatográfico das frações enriquecidas em alcaloides (teste positivo com solução de Dragendorff) dos extratos etanólicos em amônia das cascas de A. desmanthum e A. vargasii resultaram nos isolamentos e posteriores identificações dos alcaloides elipticina (1) e N-metiltetrahidroelipticina 2 de A. vargasii e aspidoscarpina (3) de A. desmanthum.

A elipticina foi identificada pela análise dos espectros de RMN de ${ }^{1} \mathrm{He} \mathrm{e}^{13} \mathrm{C}$. Analisando o espectro de $\mathrm{RMN}$ de ${ }^{1} \mathrm{H}$ de 1 mostrou em $\delta$ 7,2 a 9,5 os seguintes sinais para o núcleo piperidínico: um singleto em $\delta$ 9,5 atribuído ao $\mathrm{H}-18$, um dubleto $(J 7,0 \mathrm{~Hz})$ em $\delta 7,9$ referente ao $\mathrm{H}-14$, um dubleto $(J 7,0 \mathrm{~Hz})$ em $\delta 8,3$ e atribuído ao H-3 e, para o núcleo indólico, um dubleto $(\mathrm{J} 7,0 \mathrm{~Hz})$ para $\mathrm{H}-9$, um multipleto em $\delta$ 7,5 com integração equivalente a dois hidrogênios foi atribuído a $\mathrm{H}-11$ e $\mathrm{H}-12$, e um duplo duplo dubleto centralizado em $\delta 7,2$ que foi atribuído a $\mathrm{H}-10(J 7,5 ; 6,8 ; 1,5 \mathrm{~Hz})$.

As duas metilas de 1 foram evidenciadas por singletos em $\delta_{\mathrm{H}} 2,7 \mathrm{e}$ 3,2 e sua localização no esqueleto indoloisoquinolínico, bem como a atribuição desses sinais, foram realizadas baseando-se no espectro de HMBC (Figura 2). Nesse último, as interações a três ligações entre os hidrogênios metílicos com $\delta_{\mathrm{H}} 3,2(\mathrm{H}-21)$ e os carbonos com $\delta_{\mathrm{c}} 122,2$ $(\mathrm{C}-19)$ e $\delta_{\mathrm{c}} 124,8$ (C-7) e entre os hidrogênios metílicos com $\delta_{\mathrm{H}} 2,7$ $(\mathrm{H}-17)$ e os carbonos com $\delta_{c} 133,4(\mathrm{C}-15)$ e $\delta_{c} 141,9(\mathrm{C}-2)$ permitiram atribuição inequívoca dos sinais das metilas. Também, através das correlações no espectro de NOESY, foram evidenciadas interações através do espaço (Figura 2) entre $\mathrm{H}-21$ e os hidrogênios com $\delta_{\mathrm{H}} 8,3$ (H-9) e 9,5 (H-18) e entre H-17 e o hidrogênio com $\delta_{\mathrm{H}} 7,9$ (H-14). $\mathrm{O}$ espectro de ESI-HR-MS possibilitou a determinação da fórmula molecular do alcaloide $\mathbf{1}$ em $\mathrm{C}_{17} \mathrm{H}_{14} \mathrm{~N}_{2}$. Os dados espectrais de $\mathbf{1}$ foram também comparados com a literatura, ${ }^{9}$ permitindo identificá-lo como elipticina (1). A elipticina também foi isolada de outras espécies, como A. williamsii, ${ }^{4}$ A. dasycarpon, A. ulei,${ }^{17}$ A. subincanum,${ }^{18}$ A. gilbertii ${ }^{19}$ e Ochrosia elliptica labill. ${ }^{20}$ Nas últimas décadas tem recebido uma grande atenção, devido sua propriedade antitumoral. ${ }^{21}$ Há relatos na literatura de um grande número de trabalhos com síntese de elipticina e seus análogos..$^{22,23}$ 


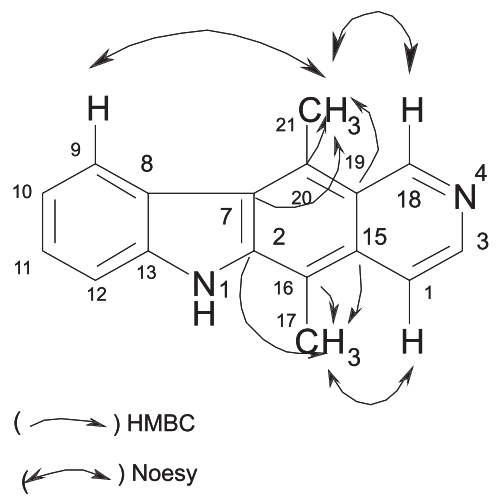

Figura 2. Correlações $\mathrm{C}$-H e interações $\mathrm{H}$-H por meio de experimentos de $H M B C$ e NOESY, respectivamente, para o alcaloide 1

O espectro de IV de 2 exibiu banda de absorção em $v_{\text {máx }} 3240 \mathrm{~cm}^{-1}$, referente à deformação axial de N-H, em $v_{\text {máx }} 2918$ e $2847 \mathrm{~cm}^{-1}$, referentes à presença de deformações axiais $\mathrm{C}$ - $\mathrm{H}$, sugerindo a presença de grupos metilas. $\mathrm{O}$ espectro de $\mathrm{RMN}$ de ${ }^{1} \mathrm{H}$ de $\mathbf{2}$ apresentou sinais de hidrogênios do anel piperidínico em $\delta 2,8(\mathrm{H}-3, \mathrm{t}, 6,5 \mathrm{~Hz}), \delta 3,0$ $(\mathrm{H}-14, \mathrm{t}, 6,5 \mathrm{~Hz}), \delta 3,8(\mathrm{H}-18, \mathrm{~s})$ e singletos em $\delta_{\mathrm{H}} 2,4,2,6$ e 2,7 referentes aos hidrogênios metílicos $\mathrm{H}-17$, H-4' e H-21, respectivamente. $\mathrm{O}$ espectro de $\mathrm{HMBC}$ revelou interações em três ligações (Figura 3) através de correlações entre os sinais em $\delta 2,7(\mathrm{H}-21)$ e os sinais de C-19 $(\delta 122,2)$ e C-7 $(\delta 120,0)$, entre os sinais em $\delta 2,4(\mathrm{H}-17)$ e os de $\mathrm{C}-2(\delta 138,7)$ e $\mathrm{C}-15(\delta 128,6)$, e entre o sinal em $\delta 2,6\left(\mathrm{H}-4^{\prime}\right)$ e os de $\mathrm{C}-18(\delta 56,5)$ e C-3 $(\delta 52,5)$, o que tornou possível estabelecer as posições das metilas nos anéis piperidínico e no núcleo 1,4 dimetilbenzeno. O espectro de NOESY também ajudou na confirmação dessas posições das metilas, podendo-se destacar interações entre H-21 e H-9 e H-18, entre H-14 e H-3 e H-17, e entre H-4' e H-3 e H-18 (Figura 3). O espectro de ESI-HR-MS permitiu a determinação da formula molecular de $\mathrm{C}_{18} \mathrm{H}_{20} \mathrm{~N}_{2}$ para 2 . Os dados espectrais foram comparados com a literatura ${ }^{10}$ e possibilitou a identificação desse alcaloide como a $\mathrm{N}$-metiltetra-hidroelipticina $\mathbf{2}$.

$\mathrm{O}$ espectro de IV de 3 exibiu bandas de absorção em $v_{\text {máx }} 1580$ e $1463 \mathrm{~cm}^{-1}$, referentes a deformações axiais das ligações $\mathrm{C}=\mathrm{C}$ do anel aromático. A banda de absorção em $753 \mathrm{~cm}^{-1}$ também evidencia a natureza aromática da substância. Bandas de absorção em $1631 \mathrm{~cm}^{-}$ ${ }^{1}$, característica de deformação axial $\mathrm{C}=\mathrm{O}$ de ligação de hidrogênio interna na carbonila amídica e em $2936 \mathrm{~cm}^{-1}$, referente à deformação axial de $\mathrm{C}-\mathrm{H}$ de grupos metilas.

$\mathrm{O}$ espectro de RMN de ${ }^{1} \mathrm{H}$ apresentou sinais na região de hidrogênios aromáticos em $\delta_{\mathrm{H}} 6,6(\mathrm{H}-9, \mathrm{~d}, 8 \mathrm{~Hz})$ e em 6,7 (H-10, d, 8 Hz), além de sinal em $\delta_{\mathrm{H}} 10,95$, consistente com a presença de uma hidroxila. $\mathrm{O}$ sinal em $\delta_{\mathrm{H}} 3,8 \mathrm{com}$ integração para três hidrogênios foi atribuído ao grupo metoxila e esse sinal correlaciona-se ao carbono metílico C-11'em $\delta 53,7$. Também, a presença de oito carbonos metilênicos em $\delta$ 53,9 (C-3), 52,7(C-5), 39,6 (C-6), 23,2 (C-14), 34,3 (C-15), 25,4 (C-16), 21,8(C-17) e 30,3 (C-19) foi revelada pela análise do espectro de DEPT 135. Pela análise do espectro de COSY (Figura 4 e Figura $3 \mathrm{~S}$, material suplementar) destacaram-se as seguintes correlações ${ }^{3} \mathrm{~J}$ : H-14' $(\delta 1,14)$ com H-3 e H-15 ( $\delta 1,9-2,1$ e $1,5-1,7$, respectivamente), H-5' $(\delta 2,3)$ com H-6' $(\delta 1,5-1,7)$, H-2 $(\delta 4,1)$ com H-16 e H-16' $(\delta$ $1,5-1,7$ e $1,8-1,9$, respectivamente) e H-17 ( $\delta 1,5-1,7)$ com H-16' $(\delta 1,8$ $1,9)$. Observou-se em $\delta 2,23$ um singleto referente ao H-21. Também, através das correlações no espectro de NOESY (Figura 4S, material suplementar), foram evidenciadas interações através do espaço entre H-21 e H-15 $\left(\delta_{\mathrm{H}} 1,5-1,7\right)$, H-5 $\left(\delta_{\mathrm{H}} 3,1\right), \mathrm{H}-3\left(\delta_{\mathrm{H}} 3,0\right)$ e H-9 $\left(\delta_{\mathrm{H}} 6,6\right)$. $\mathrm{O}$ espectro de ESI-HR-MS possibilitou a determinação da fórmula molecular do alcaloide $3 \mathrm{em} \mathrm{C}_{22} \mathrm{H}_{30} \mathrm{~N}_{2} \mathrm{O}_{3}$. A estrutura do alcaloide 3 foi estabelecida com base nos dados dos espectros de RMN de ${ }^{1} \mathrm{H}$ e de ${ }^{13} \mathrm{C}$
(Figuras 1S e 2S, material suplementar), massas, bem como através de comparação com valores dos seus deslocamentos químicos na literatu$\mathrm{ra}^{24}$ e possibilitou a identificação do alcaloide 3 como aspidoscarpina, a qual foi isolada também das cascas de A. marcgravianum, ${ }^{25}$ A. limae, ${ }^{26}$ A. formasanum. ${ }^{27} \mathrm{~A}$ aspidoscarpina (3) mostrou-se bastante ativa in vitro frente à linhagem K1 de Plasmodium falciparum. ${ }^{5}$

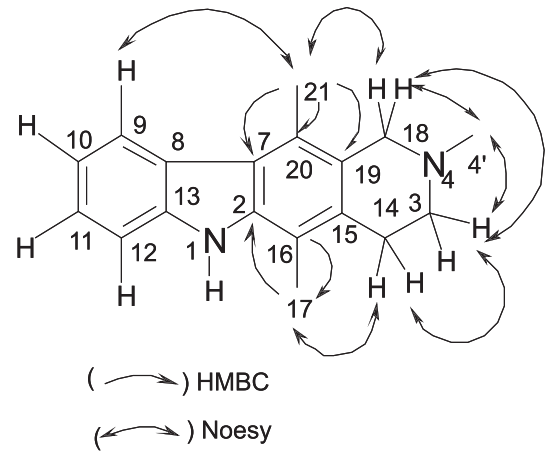

Figura 3. Correlações $\mathrm{C}$-H e interações $\mathrm{H}$ - $\mathrm{H}$ por meio de experimentos de HMBC e NOESY, respectivamente, para o alcaloide 2

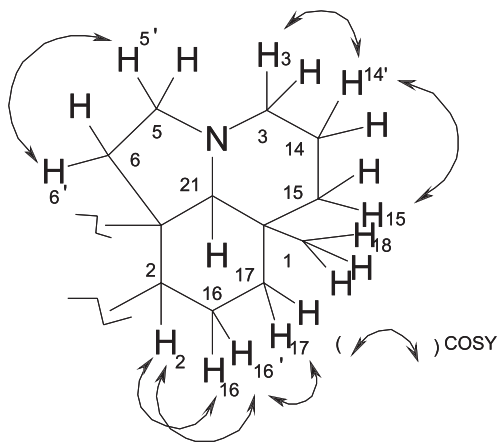

Figura 4. Correlações H-H por meio de experimento de COSY para o alcaloide 3

\section{CONCLUSÃO}

Os extratos alcaloídicos de A. vargasii e A. desmanthum mostraramse ser uma fonte promissora de alcaloides indólicos, dos quais foram isolados elipticina, N-metiltetra-hidroelipticina e aspidoscarpina. A continuação dos estudos dessas espécies torna-se necessária, devido essa classe de alcaloides possuir um espectro de atividades biológicas comprovadas. Testes para atividade antimalárica realizados com os alcaloides elipticina (1) e aspidoscarpina (3) confirmaram atividade em $P$. falciparum. ${ }^{5}$ Os resultados obtidos neste estudo confirmam a importância da investigação química e biológica das espécies de Aspidosperma na busca de novos agentes biologicamente ativos.

\section{MATERIAL SUPLEMENTAR}

No material suplementar, disponível gratuitamente em http:// quimicanova.sbq.org.br, na forma de PDF, estão apresentados os espectros de RMN de ${ }^{1} \mathrm{H}$ (Figura 1S) e de ${ }^{13} \mathrm{C}$ (Figura 2S), COSY (Figura 3S) e Noesy (Figura 4S) do alcaloide aspidoscarpina (3) utilizados na sua identificação estrutural.

\section{AGRADECIMENTOS}

Ao CNPq (PNOPG 520.354/99-0, 550.260/01-3; PPBIO/MCT 480.002/04-5; PPG-7 557.106/05-2) e PROBEM (Contrato Bioamazonia-BASA-FEPAD) pelos financiamentos recebidos. Ao J. C. Tomaz, Dr. C. Carollo e Prof. N. Lopes (FCFRP/USP) pelas análises 
de HR-ESI-MS. Ao Dr. M. Yoshida, L. C. Oliveira e L. C. Roque do CBA (Manaus-AM) pelos espectros de RMN. Ao Prof. V. F. Veiga Jr. pela leitura crítica desse manuscrito.

\section{REFERÊNCIAS}

1. Endress, M. E.; Bruyns, P. V.; The Botanical Review 2000, 66, 1; Pereira, M. M.; Jácome, R. L. R. P.; Alcântara, A. F. de C.; Alves, R. B.; Raslan, D. S.; Quim. Nova 2007, 30, 970.

2. Marcondes-Ferreira, W.; Kinoshita, L. S.; Rev. Bras. Bot. 1996, 19, 203.

3. Bolzani, W. S.; Serur, L. M.; Matos, F. J. A.; Gottlieb, O. R.; Biochem. Syst. Ecol. 1987, 15, 187.

4. Sakamoto-Hojo, E. T.; Takahashi, C. S.; Ferrari, I.; Motidome, M.; Mutat. Res. 1988, 1, 11.

5. Mitaine, A. C.; Weniger, B.; Sauvain, M.; Lucumi, E.; Aragón, R.; Zèches-Hanrot, M.; Planta Med. 1998, 64, 487; Andrade Neto, V. F.; Pohlit, A. M.; Pinto, A. C. S.; Silva, E. C. C.; Nogueira, K. L.; Melo, M. R. S.; Henrique, M. C.; Amorim, R. C. N.; Silva, L. F. R.; Costa, M. R. F.; Nunomura, R. C. S.; Nunomura, S. M.; Alecrim, W. D.; Alecrim, M. G. C.; Mem. Inst. Oswaldo Cruz 2007, 102, 359.

6. Verpoorte, R.; Kos-Kuyck, E.; Tjin A Tsoi, A.; Ruigrok, C.L.M.; de Jong, G.; Svendsen, A. B.; J. Med. Plant. Res. 1983, 48, 289

7. Verpoorte, R.; Ruigrok, C. L. M.; Svendsen, A. B.; J. Med. Plant. Res. 1982a, 46, 149.

8. Pedersen, J. M.; Bowman, W. R.; Elsegood, M. R. J.; Fletcher, A. J.; Lovell, P. J.; J. Org. Chem. 2005, 70, 10615.

9. Ishikura, M.; Hino, A.; Yaginuma, T.; Agata, I.; Katagiri, O.; Tetrahedron 2000, 56, 193; Ahond, A.; Popat, C.; Potier, P.; Tetrahedron 1978, 34, 2385.

10. Burnell, R. H.; Casa, D. D.; Can. J. Chem. 1967, 45, 89; Azoug, M.; Loukaci, A.; Richard, B.; Nuzillard, J.-M.; Zeches-Hanrot, M.; Le Men-Olivier, L.; Moreti, C.; Phytochemistry 1995, 39, 1223.

11. Gilbert, B.; Duarte, A. P.; Nakagawa, Y.; Joule, J. A.; Flores, S. E.; Briolesse, J. A.; Campello, J.; Carrazzoni, E. P.; Owellen, R. J.; Blossey, E. C.; Brown Jr., K. S. E.; Djerassi, C.; Tetrahedron 1965, 21, 1441; Mclean, S.; Palmer, K.; Marion, L.; Can. J. Chem. 1960, 38, 1547.
12. Oliveira, F. Q.; Junqueira, R. G.; Stehmann, J. R.; Brandão, M. G. L.; Rev.Bras. Plant. Med. 2003, 5, 23.

13. Bourdy, G.; Oporto, P.; Gimenez, A.; Deharo, E.; J. Ethnopharmacol. 2004, 93, 269

14. Ribeiro, J. E. L. S.; Hopkins, M. J. G; Vicentini, A.; Sothers, C. A.; Costa, G.; Assunção, P. A. C. L.; Pereira, E. C.; Mesquita, M. R.; Procópio, L. C.; Flora da Reserva Ducke: Guia de Identificação das Plantas Vasculares de uma Floresta de Terra-Firme na Amazônia Central, INPA: Manaus, 1999.

15. Ferreira, I. C. P.; Lonardoni, M. V. C.; Machado, G. M. C.; Leon, L. L.;Gobbi-Filho, L.; Pinto, L. H. B.; Oliveira, A. J. B.; Mem. Inst. Oswaldo Cruz, 2004, 99, 325.

16. Mitaine, A. C.; Weniger, B.; Sauvain, M.; Lucumi, E.; Aragón, ???; Zèches-Hanrot, M.; Planta Med. 1998, 65, 487.

17. Pereira, M. M.; Jácome, R. L. R. P.; Alcântara, A. F.de C.; Alves, R. B.; Raslan, D. S.; Quim. Nova 2007, 30, 970.

18. Büchi, G.; Warnhoff, E. W.; J. Am. Chem. Soc. 1959, 81, 4434.

19. Duarte, A. P.; Miranda, E. C.; An. Acad. Bras. Cienc. 1983, 55, 189.

20. Kuroda, M.; Mimaki, Y.; Kawase, N.; Yokosura, A.; Sashida, Y.; Kagiya, A.; Natural Medicines 1999, 53, 272.

21. Paoletti, C.; Lesca, C.; Cros, S.; Malvy, C.; Auclair, C.; Biochem. Pharmacol. 1979, 28, 345.

22. Zhang, Q.; Shi, C.; Zhang, H. R.; Wang, K. K.; J. Org. Chem. 2000, 65, 7977.

23. Bergman, J.; Pelcman, B.; Tetrahedron 1988, 44, 5215.

24. Mclean, S.; Reynolds, W. F.; Zhu, X.; Can. J. Chem. 1987, 65, 200.

25. Arndt, R. R.; Brown, S. H.; Ling, N. C.; Roller, P.; Djerassi, C.; FerreiraFilho, J. M.; Gilbert, B.; Miranda, E. C.; Flores, S. E.; Duarte, A. P.; Carrazzoni, E. P.; Phytochemistry 1967, 6, 1653.

26. Pinar, M.; Bycroft, B. W.; Seibl, J.; Schmid, H.; Helv. Chim. Acta 1965 , 48,822 .

27. M. Garcia, R. F.; Brown, K. S.; Phytochemistry 1976, 15, 1093. 


\section{ALCALOIDES INDÓLICOS DE CASCAS DE Aspidosperma vargasii E A. desmanthum}

\section{Marycleuma Campos Henrique}

Departamento de Química, Instituto de Ciências Exatas, Universidade Federal do Amazonas, Av. Rodrigo Octávio Jordão Ramos, 3000, 69077-000 Manaus - AM, Brasil

\section{Sergio Massayoshi Nunomura e Adrian Martin Pohlit*}

Coordenação de Pesquisa em Produtos Naturais, Instituto Nacional de Pesquisa da Amazônia, Av. André Araújo, 2936, 69060-001 Manaus - AM, Brasil

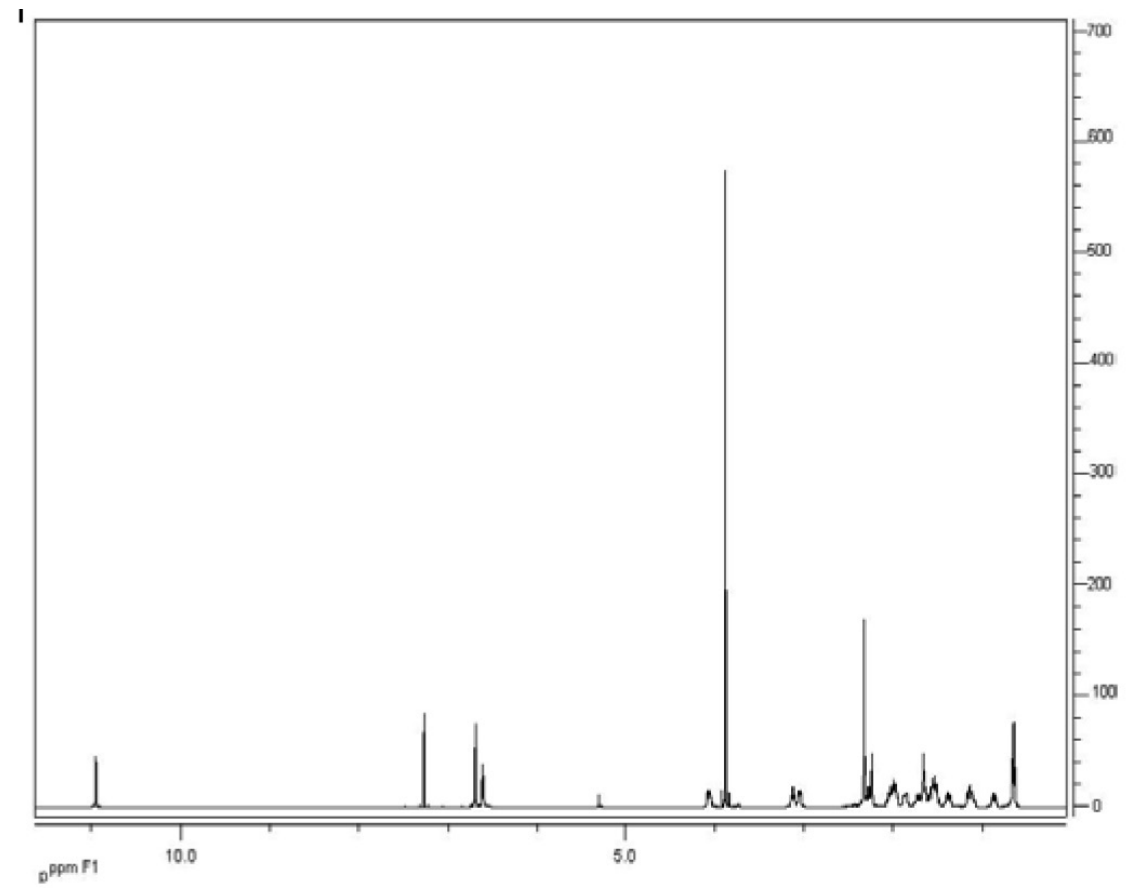

Figura 1S. Espectro de $\mathrm{RMN}$ de ${ }^{1} \mathrm{H}$ de $500 \mathrm{MHz}_{\text {em }} \mathrm{CDCl}_{3}$ do alcaloide 3

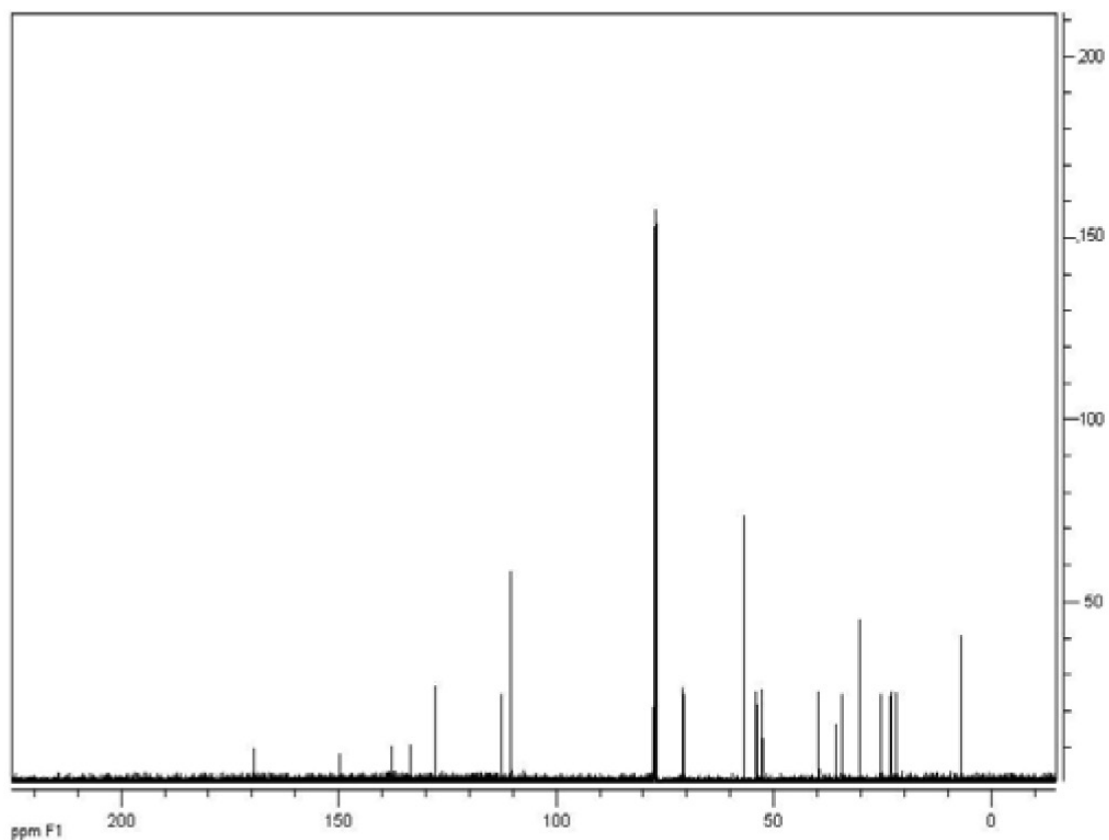

Figura 2S. Espectro de $\mathrm{RMN}$ de ${ }^{13} \mathrm{C}$ de $125 \mathrm{MHz}_{\text {em }} \mathrm{CDCl}_{3}$ do alcaloide 3

*e-mail: ampohlit@inpa.gov.br 


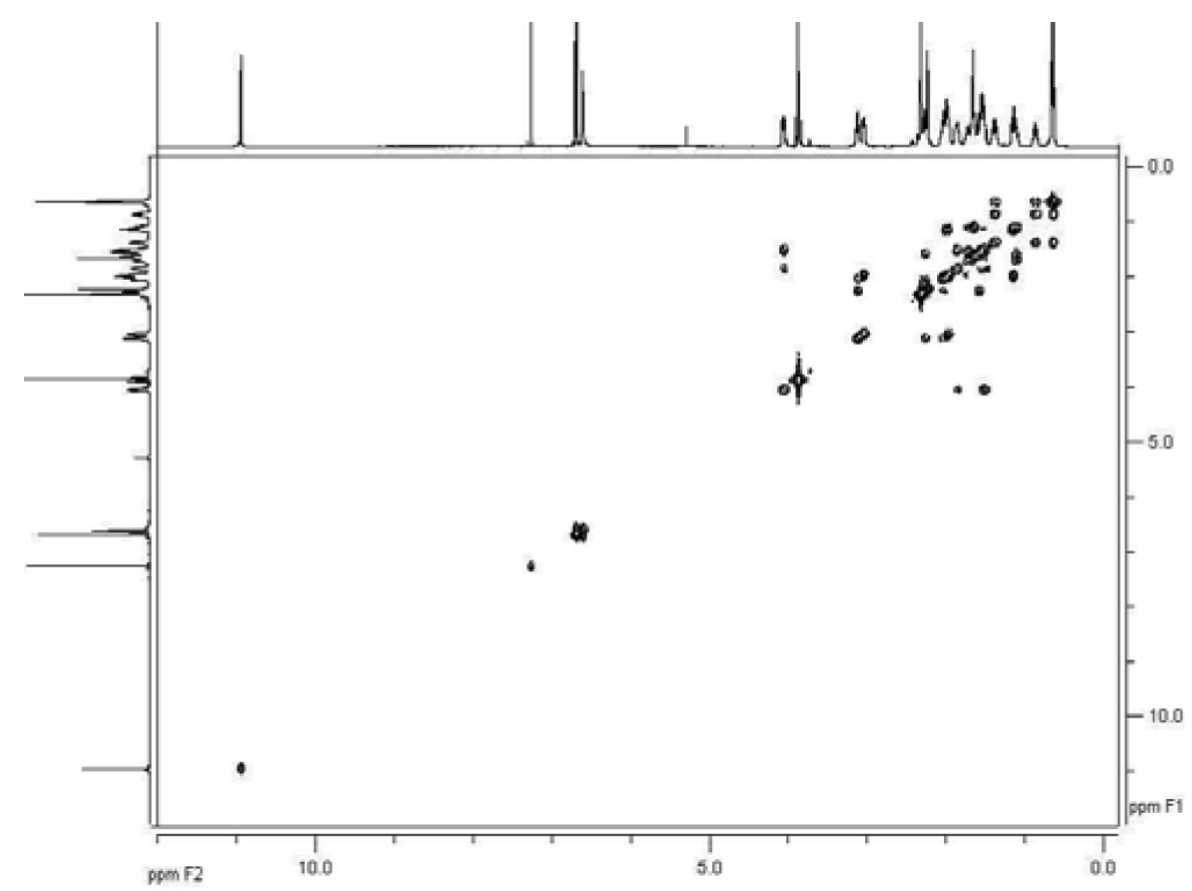

Figura 3S. Espectro de COSY em $\mathrm{CDCl}_{3}$ do alcaloide 3

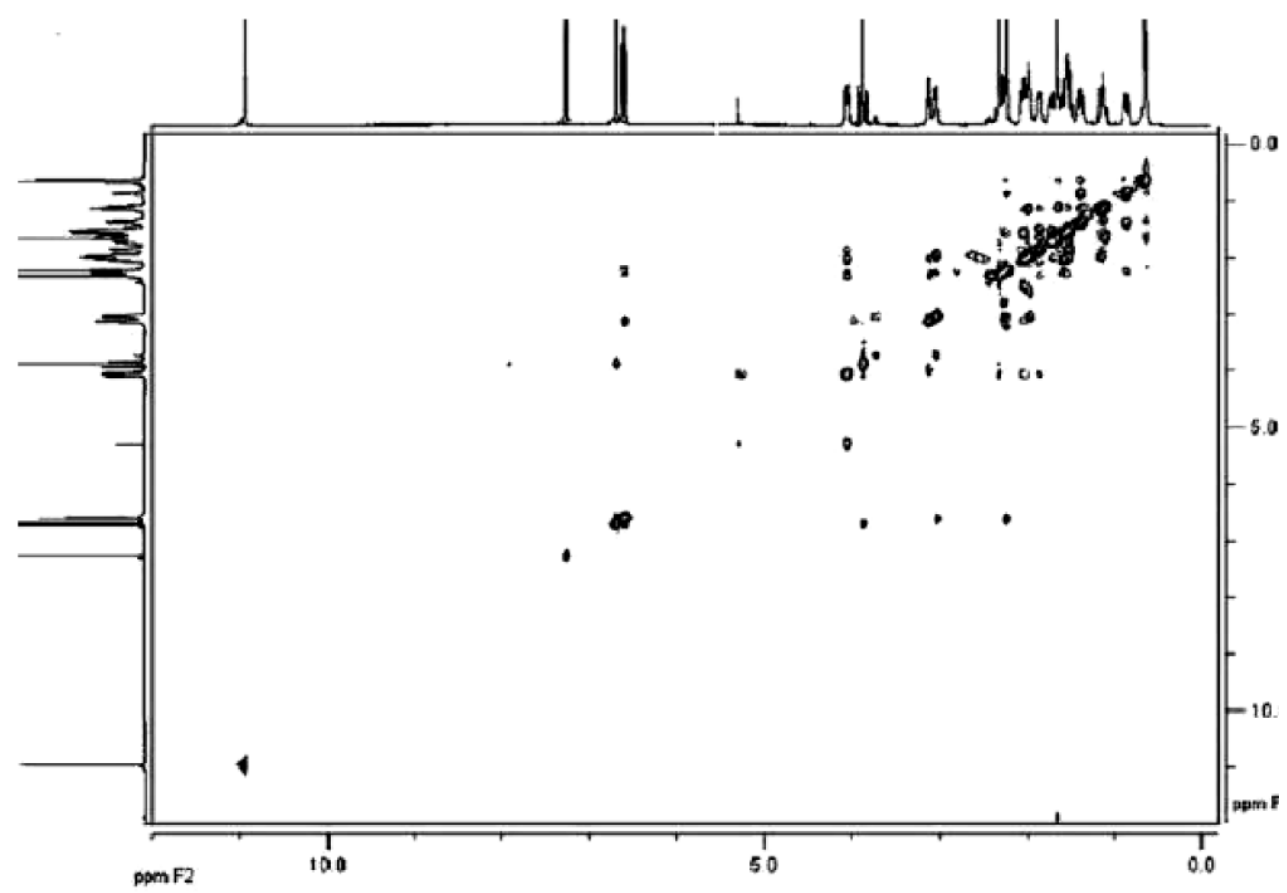

Figura 4S. Espectro de NOESY em $\mathrm{CDCl}_{3}$ do alcaloide 3 